\title{
Impact of pressure anisotropy on tokamak equilibria and the toroidal magnetic precession
}

\author{
M Jucker ${ }^{1}$, J P Graves ${ }^{1}$, G A Cooper ${ }^{1,2}$ and W A Cooper ${ }^{1}$ \\ ${ }^{1}$ Ecole Polytechnique Fédérale de Lausanne (EPFL), Centre de Recherches en \\ Physique des Plasmas, Association EURATOM-Confédération Suisse, CH-1015 \\ Lausanne, Switzerland \\ E-mail: martin.jucker@epfl.ch \\ ${ }^{2}$ Department of Physics, University of the South, Sewanee, Tennessee 37383, USA
}

\begin{abstract}
Using a generalised anisotropic tokamak equilibrium and an exact guiding centre drift formulation, the effect of parallel and perpendicular anisotropy on the toroidal precession drift is investigated. Significant differences between parallel and perpendicular pressure anisotropy are observed. While the Shafranov shift is not sensitive to the ratio of the parallel and perpendicular pressures $p_{\perp} / p_{\|}$, the deepening of the magnetic well is found to be sensitive to $p_{\perp} / p_{\|}$. Here, the diamagnetic effect identified by Connor et al. [J. Connor, R. Hastie, and T. Martin, Nucl. Fusion 23, 1702 (1983)] is generalised and found to depend crucially on the deposition of the energetic ions on which the equilibrium depends, and leads to test particle precessional drifts that depend sensitively on pitch angle.
\end{abstract}

PACS numbers: 52.20.Dq, 52.35.Py, 52.55.Fa, 52.65.Cc 


\section{Introduction}

Regions of unfavourable curvature can lead to instabilities such as collisionless trappedelectron modes, fishbones and many others. The stability of these modes is largely determined by the toroidal precession drift of trapped particles. Rosenbluth and Sloan [1] have shown that such modes can be stabilised in regions of bad curvature through plasma diamagnetism (i.e. the effect of the pressure gradient on the magnetic field strength), which can inverse the sign of the precession drift frequency $\omega_{D}$ of a single particle. Furthermore, Beer et al. [2] have shown the importance of the toroidal drift on trapped electron modes using a $s-\alpha$ model and concentrated on the effects of the local and global shear on $\omega_{D}$ in enhanced reverse-shear discharges, where it was found that the dominant stabilising effect was the reversal of the toroidal precession drifts of barely trapped electrons. We note here that the local shear is defined by the second derivative of the Shafranov shift. Furthermore, a few years earlier, Wu et al. [3] studied the effects of trapped alpha particles on internal kink and fishbone modes, including shaping, local and global shear. It was found that elongation and the Shafranov shift can significantly reduce the magnitude of the precessional drift frequency and enhance the trapped particle drift reversal domain in pitch angle space. Finally, Connor et al. [4] investigated the effects of pressure gradients self-consistently by considering the diamagnetic well effect together with the effect of local and global shear. The latter study is nevertheless essentially restricted to equilibria which do not contain a significant auxiliary heated fast ions energy content. Indeed, the authors stress that a consistent treatment of the effect of finite $\beta$ from energetic ions on the toroidal precession drift involves an anisotropic pressure equilibrium.

In the present article, we will consider the precession drift of particles in a realistic tokamak equilibrium including full shaping, shear and pressure profiles, and will concentrate on the effects of pressure anisotropy, both parallel dominated and perpendicular. Clearly this is also motivated by the fact that ICRH heating creates anisotropic equilibria where $p_{\perp}>p_{\|}$, while NBI heating typically has parallel anisotropy $p_{\perp}<p_{\|}$. Deposition can be strongly localised, creating large gradients in local beta values $\left(\beta=2 p / B^{2}\right)$, which in turn gives rise to a reduction or even reversal in the toroidal drift precession.

The article is organised as follows: In section 2, the equilibria are described and the guiding centre drift orbit equations are given explicitely. Section 3 shows the resulting effects of pressure, including anisotropy, on the toroidal precession drift frequency and section 4 is dedicated to the deepening of the poloidal magnetic well in perpendicularly anisotropic equilibria and tight aspect ratio. 


\section{Equilibrium and orbit equations}

\subsection{Anisotropic equilibrium}

VMEC [5] and TERPSICHORE [6] have been adapted to encompass pressure anisotropy [7] in the equilibrium. A modified bi-Maxwellian [8] is used for hot particles in order to prescribe different pressure profiles parallel and perpendicular to the magnetic field. It has recently been shown that such a bi-Maxwellian can be adequate for describing some features of ICRH heating [9]. The new form of the codes also incorporates a new parameter $B_{c}$, which indicates the peak of the hot particle profile and thus the location of the resonant heating layer. The distribution reads:

$$
F_{h}(\psi, E, \mu)=\left(\frac{m}{2 \pi}\right)^{3 / 2} \frac{n_{c}(\psi)}{T_{\perp}(\psi) T_{\|}^{1 / 2}(\psi)} \exp \left[-\frac{\mu B_{c}}{T_{\perp}(\psi)}-\frac{\left|E-\mu B_{c}\right|}{T_{\|}(\psi)}\right],
$$

where $E$ denotes the particle energy, $m$ the mass and $\mu=m v_{\perp}^{2} / 2 B$ the magnetic moment. $\psi$ is a flux label. The parallel and perpendicular temperatures are $T_{\|}$and $T_{\perp}$ respectively. Explicit calculation of the second moments of the above distribution function yields expressions for the parallel as well as perpendicular pressures $p_{\|}, p_{\perp}$ :

$$
p_{\|}=n_{c} T_{\|} H_{\|} \text {and } p_{\perp}=n_{c} T_{\perp} H_{\perp},
$$

where for $B>B_{c}$ :

$$
H_{\|}=\left(\frac{T_{\perp a}}{T_{\perp}}\right) \text { and } H_{\perp}=\left(\frac{T_{\perp a}}{T_{\perp}}\right)^{2}
$$

while for $B<B_{c}$ :

$$
\begin{aligned}
H_{\|}= & {\left[\frac{T_{\perp a}}{T_{\perp}}+\left(\frac{T_{\perp}}{T_{\|}}\right)^{3 / 2}\left(\frac{B_{c}-B}{B_{c}}\right)^{3 / 2}\left(\frac{T_{\perp b}-T_{\perp a}}{T_{\perp}}\right)\right] } \\
H_{\perp}= & {\left[\left(\frac{T_{\perp a}}{T_{\perp}}\right)^{2}+\right.} \\
& \left.\left(\frac{T_{\perp}}{T_{\|}}\right)^{1 / 2}\left(\frac{B_{c}-B}{B_{c}}\right)^{1 / 2}\left\{\frac{T_{\perp b}-T_{\perp a}}{2 T_{\perp}}\left(\frac{B}{B_{c}}\right)+\frac{T_{\perp b}^{2}-T_{\perp a}^{2}}{T_{\perp}^{2}}\right\}\right] .
\end{aligned}
$$

Here,

$$
T_{\perp a}=T_{\perp}\left[\frac{B_{c}}{B}+\frac{T_{\perp}}{T_{\|}}\left(1-\frac{B_{c}}{B}\right)\right]^{-1}
$$

and

$$
T_{\perp b}=T_{\perp}\left[\frac{B_{c}}{B}-\frac{T_{\perp}}{T_{\|}}\left(1-\frac{B_{c}}{B}\right)\right]^{-1},
$$

and $B_{c}$ is a function of $\psi$ only (though usually a constant), and $B$ is a function of $\psi$ and $\theta$ in a tokamak. One can see that the appearance of the anisotropy factor $T_{\perp} / T_{\|}$in $(4 a)$ and $(4 b)$ is such that the poloidal dependence of the magnetic field is translated into poloidal pressure dependence when $T_{\perp} / T_{\|}>1$ (and here $p_{\perp} / p_{\|}>1$, i.e. perpendicular pressure anisotropy). This means amongst other things that the pressure is no longer 


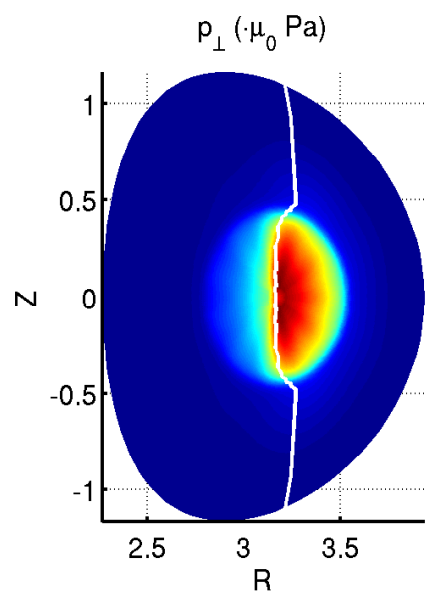

(a) $p_{\perp}$ in the RZ plane.

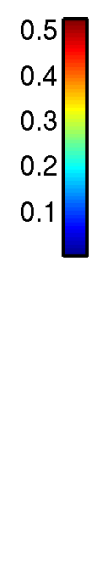

0.3
0.2
0.1

Figure 1. Perpendicular pressure in a JET like anisotropic equilibrium $\left(T_{\perp} / T_{\|}=10\right)$. The pressure contains a poloidal dependence due to pressure anisotropy. The white line denotes the locations where $B=B_{c}$.

a flux surface quantity. In the other case, $T_{\perp} / T_{\|}<1$, the poloidal dependence in the pressure is much weaker, as has been stated earlier in the analytic work of Ref. [10]. Figure 1 shows the pressure distribution of an equilibrium with perpendicular anisotropy, $T_{\perp} / T_{\|}=10$, where one can clearly see the poloidal dependence of $p_{\perp}(\psi, \theta)$.

We know [4] that in isotropic plasmas the effect of the pressure on the precession drift frequency enters through a local shear and a magnetic well effect. The local shear is proportional to $r \Delta^{\prime \prime}$, where $\Delta$ denotes the Shafranov shift defined through the relation $R=R_{0}-\Delta(r)+r \cos \theta$, where $R_{0}$ is the radius of the magnetic axis, and $r$ is the local minor radius. The magnetic well effect in an isotropic plasma is described through the ballooning mode parameter

$$
\alpha=-\frac{2 R_{0} q^{2}}{B_{0}^{2}} \frac{d p}{d r}
$$

The radial variable $r$ is in the present work numerically mapped against the toroidal flux approximately given by $r^{2} / a^{2}$, with $a$ the edge minor radius. Also, $B_{0}$ is the magnetic field strength on axis and $q$ the safety factor. The new version of VMEC employed here demonstrates that $\Delta^{\prime \prime}, \Delta^{\prime}$ and $\Delta$ compare well with Ref. [10] where it was stated that $p_{\perp}+p_{\|}$essentially determines $\Delta$. In contrast, the magnetic well effect depends entirely on $p_{\perp}$ through the relation for the toroidal field

$$
B_{T}=\frac{g}{\sigma R},
$$

where $g=B_{0} R_{0}\left(1+g_{2}\right)$ and in large aspect ratio tokamaks,

$$
\frac{\partial}{\partial r}\left(\frac{g_{2}}{\sigma}\right)=-\frac{1}{R_{0}}\left[\frac{\epsilon}{q^{2}}(2-s)+\frac{R_{0}}{B_{0}^{2}} \frac{\partial p_{\perp}}{\partial r}\right] .
$$

Here, $\epsilon=r / R_{0}$ and $s=(r / q) d q / d r$ denotes the magnetic shear. As pointed out earlier, in isotropic plasmas $\alpha$ plays an important role in the stabilisation of curvaturetype microinstabilities [11, 12]. In fact, as shown in Refs. [1] and [4], high $\alpha$ lowers 
the magnetic precession drift frequency and can even invert its sign, which suppresses the above mentioned microinstabilities [12]. Nevertheless, in an anisotropic plasma it is now clear that the perpendicular and parallel pressure will have different impacts on the equilibrium, and we will show here that it is indeed the perpendicular component of the pressure tensor, and its poloidal variation, which determines the role of the diamagnetic effects on single particle orbits.

Given Eqs. (7) and (8) it is clearly convenient in this study to define a new parameter

$$
\alpha_{\perp}=-\frac{2 R_{0} q^{2}}{B_{0}^{2}} \frac{\partial p_{\perp}}{\partial r}
$$

thus depending only on the perpendicular pressure component. Note that in the isotropic limit, $\alpha=\alpha_{\perp}$, since $p_{\|}=p_{\perp}=p$.

\subsection{Guiding centre drift orbit equations}

Anisotropic equilibria give rise to new terms in the Hamiltonian description of the guiding centre drift motion. The equations of motion corresponding to an anisotropic equilibrium have been derived in Ref. [13] and are written in terms of

$$
\sigma \equiv 1-\left.\frac{1}{B} \frac{\partial p_{\|}}{\partial B}\right|_{\psi}=1-\frac{\left(p_{\|}-p_{\perp}\right)}{B^{2}}
$$

and

$$
\left.\tau \equiv \frac{\partial(\sigma B)}{\partial B}\right|_{\psi}=1+\left.\frac{1}{B} \frac{\partial p_{\perp}}{\partial B}\right|_{\psi}
$$

These two parameters are a measure of anisotropy, with the isotropic limit being identified as $\sigma=\tau=1$. Stability against the firehose criterion and the mirror criterion is assured providing $\sigma>0$ and $\tau>0$ respectively [14].

The covariant form of the magnetic field in Boozer coordinates $(\psi, \Theta, \phi)$ can be written as [13]

$$
\sigma \mathbf{B}=I(\psi) \nabla \Theta+g(\psi) \nabla \phi+g(\psi) \delta(\psi, \Theta) \nabla \psi
$$

Defining the momentum of a guiding centre particle along the magnetic field lines of the equilibrium state $P_{\|}=m v_{\|}$, we can write the Hamiltonian without perturbed fields in the form

$$
H=\gamma m_{0} c^{2}
$$

Here, $m_{0}$ is the particle rest mass, $e$ its charge and $c$ the speed of light. The relativistic factor can be written as

$$
\gamma=\sqrt{1+\frac{2 \mu}{m_{0} c^{2}} B+\frac{P_{\|}^{2}}{m_{o}^{2} c^{2}}} .
$$

From this, new exact canonical equations of motion have been derived in Ref. [15] and introduced into the single particle orbit code VENUS [16]. These allow for a 
more precise calculation of the orbits, especially in tight aspect ratio tokamaks. The equations of motion are in terms of the Boozer coordinates and the parallel Larmour radius $\rho_{\|}=m v_{\|} / e \sigma B$ and read $[15]$

$$
\begin{aligned}
\dot{\psi}= & -\left.\frac{g(\psi)}{\gamma D}\left(\frac{\mu}{e}+\sigma \tau \frac{e B}{m} \rho_{\|}^{2}\right) \frac{\partial B}{\partial \Theta}\right|_{\psi} \\
\dot{\Theta}= & \frac{g(\psi)}{\gamma D}\left[\left.\left(\frac{\mu}{e}+\sigma \tau \frac{e B}{m} \rho_{\|}^{2}\right) \frac{\partial B}{\partial \psi}\right|_{\Theta}+\left.\frac{e B^{2} \sigma}{m_{0}} \rho_{\|}^{2} \frac{\partial \sigma}{\partial \psi}\right|_{\Theta}\right] \\
& +\frac{e \sigma^{2} B^{2}}{\gamma m_{0} D} \rho_{\|}\left(1-\rho_{\|} \frac{d g(\psi)}{d \psi}\right) \\
\dot{\phi}= & -\frac{I(\psi)}{\gamma D}\left[\left.\left(\frac{\mu}{e}+\sigma \tau \frac{e B}{m} \rho_{\|}^{2}\right) \frac{\partial B}{\partial \psi}\right|_{\Theta}+\left.\frac{e B^{2} \sigma}{m_{0}} \rho_{\|}^{2} \frac{\partial \sigma}{\partial \psi}\right|_{\Theta}\right] \\
& +\frac{e \sigma^{2} B^{2}}{\gamma m_{0} D} \rho_{\|}\left(q(\psi)+\rho_{\|} \frac{d I(\psi)}{d \psi}\right) \\
& -\left.\frac{e \sigma^{2} B^{2}}{\gamma m_{0} D} \rho_{\|}^{2} g(\psi) \frac{\partial \delta(\psi, \Theta)}{\partial \Theta}\right|_{\psi}+\frac{g(\psi) \delta(\psi, \Theta)}{\gamma D}\left(\frac{\mu}{e}+\sigma \tau \frac{e B}{m} \rho_{\|}^{2}\right) \frac{\partial B}{\partial \Theta}(15 c) \\
\dot{\rho}_{\|}= & -\frac{1}{\gamma D}\left(1-\rho_{\|} \frac{d g(\psi)}{d \psi}\right)\left(\frac{\mu}{e}+\sigma \tau \frac{e B}{m} \rho_{\|}^{2}\right) \frac{\partial B}{\partial \Theta}
\end{aligned}
$$

In Eqs. $(15 a)-(15 d) q(\psi)$ is the safety factor and

$$
D=g(\psi) q(\psi)+I(\psi)+\rho_{\|}\left[g(\psi) \frac{d I(\psi)}{d \psi}-\frac{d g(\psi)}{d \psi} I(\psi)-g^{2}(\psi) \partial_{\Theta} \delta(\psi, \Theta)\right] .
$$

Additional terms to those contained in the conventional system of equations in e.g. Ref. [17] make the orbits exactly canonical [15]. They are proportional to $g(\psi) \delta(\psi, \Theta)$ and its derivatives, and are written separately as last terms within each equation. Note however that all the equations have additional corrections in the generalised expression for $D$ given above.

The orbits can now exactly be identified with the guiding centre velocity equation satisfying Liouville's theorem [18]

$$
\mathbf{v}_{g}=\frac{e \rho \sigma\left[\mathbf{B}+\nabla \times\left(\rho_{\|} \sigma \mathbf{B}\right)\right]}{\gamma m_{0}\left(1+\rho_{\|} \mu_{0} \mathbf{K} \cdot \mathbf{B} / B^{2}\right)},
$$

with $\mathbf{K}=\nabla \times(\sigma \mathbf{B})$ the effective current as described in Ref. [15]. In order to demonstrate the role of the corrections in $(15 a)-(15 d)$, and exhibit typical trapped drift orbits in three dimensions, Fig. 2 shows a test case of a tight aspect ratio tokamak with $R_{0}=1.1 \mathrm{~m}, a=0.9 \mathrm{~m}, B_{0}=5.6 T, \kappa_{a}=2.5$, volume averaged $\beta$ of $2 \%$ and the particle energy was set to $500 \mathrm{keV}$. Shown are the orbits of the same particle (same initial conditions and equilibrium), one without the new terms (blue) and one including them (red). One can see that the difference can be important, as the orbits are different on top and bottom of the banana orbits. 


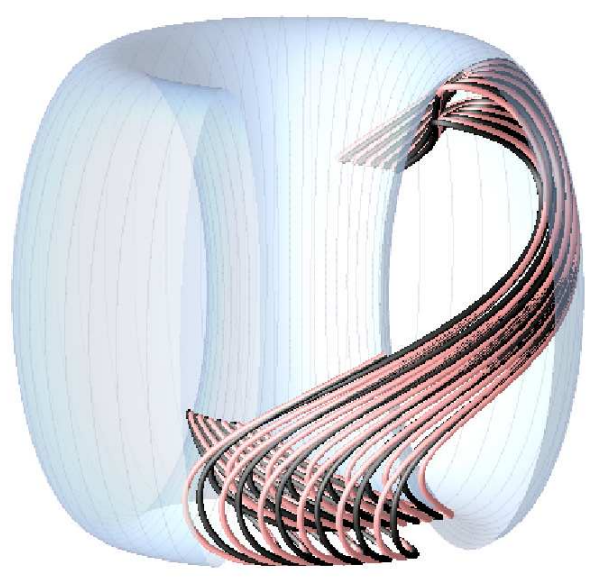

(a) $3 \mathrm{D}$ view of several banana orbits.

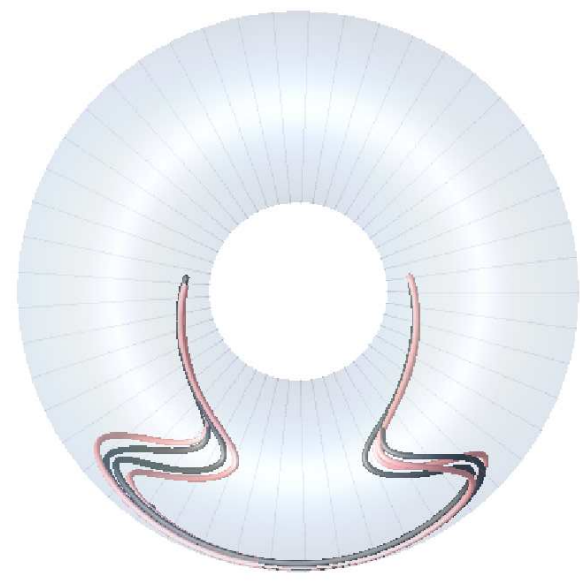

(b) Top view. For clarity, only one banana orbit is shown.

Figure 2. The additional terms in the equations of motion can contribute significantly to the single particle orbits in tight aspect ratio tokamaks. The orbits without the additional terms are plotted in blue, including the $g(\psi) \delta(\psi, \Theta)$ corrections in red.

\section{The toroidal magnetic drift frequency}

In this section, we will concentrate on the toroidal precession drifts of trapped particles. In particular, we will show the general effects of the local magnetic shear (local in the sense of the poloidal variation of the magnetic winding index [4]), which is related to $r \Delta^{\prime \prime}$ and the diamagnetic effect, which is related to $\alpha_{\perp}$. We will first investigate this analytically, and then in detail numerically.

We write the toroidal precession as

$$
\langle\dot{\phi}\rangle=\tau_{b}^{-1} \int_{0}^{\tau_{b}} d t \dot{\phi}
$$

with $\tau_{b}$ the transit/bounce time for passing/trapped particles, defined as the time it takes to complete one closed orbit in the RZ plane. Employing $d t=d \Theta / \dot{\Theta}$ we have

$$
\langle\dot{\phi}\rangle=\tau_{b}^{-1} \oint d \Theta \frac{\dot{\phi}}{\dot{\Theta}} \quad \text { with } \quad \tau_{b}^{-1}=\oint \frac{d \Theta}{\dot{\Theta}} .
$$

Expanding now $\dot{\phi} / \dot{\Theta}$ up to first order in terms forming combinations of $R_{0}\left(\Omega_{c} v_{\|}\right)^{-1}\left(v_{\perp}^{2}, v_{\|}^{2}\right)\left(B \partial / \partial \psi, R_{0}^{-2} \partial / \partial \Theta\right)$ (i.e. first order in the ratio of transit velocities and drift velocities, with $\Omega_{c}=e Z B_{0} / m$ the cyclotron frequency) we have from Eqs. $(15 a)$ and $(15 b)$

$$
\begin{aligned}
\langle\dot{\phi}\rangle=\tau_{b}^{-1} \oint d \Theta & \left\{q-\left.\frac{g q+I}{v_{\|} \Omega_{c} \sigma^{2}}\left(\frac{\mu \sigma}{m}+\frac{v_{\|}^{2} \tau}{\sigma}\right) \frac{\partial B}{\partial \psi}\right|_{\Theta}-\left.\frac{g q+I}{\Omega_{c} \sigma^{2}} v_{\|} \frac{\partial \sigma}{\partial \psi}\right|_{\Theta}\right. \\
+ & \left.\frac{v_{\|}}{\Omega_{c}}\left(\frac{d I}{d \psi}+q \frac{d g}{d \psi}\right)-\frac{g \delta}{\Omega_{c} \sigma}\left[\frac{v_{\|}}{\delta} \frac{\partial \delta}{\partial \Theta}-\left.\left(\frac{v_{\|} \tau}{\sigma^{2}}+\frac{\mu B}{m \sigma v_{\|}}\right) \frac{1}{B} \frac{\partial B}{\partial \Theta}\right|_{\psi}\right]\right\} .
\end{aligned}
$$


Now, in a large aspect ratio tokamak with circular cross section we identify $\partial / \partial \psi=$ $\left(q / r B_{0}\right) \partial / \partial r$ and to leading order in $\epsilon[10]$ :

$$
I=\frac{B_{0} r^{2}}{q R_{0}}, g=B_{0} R_{0}\left(1+g_{2}\right) \text { and } \frac{d g_{2}}{d r}=-\frac{1}{R_{0}}\left[\frac{\epsilon}{q^{2}}(2-s)+\frac{R_{0}{\overline{p_{\|}}}^{\prime}}{B_{0}^{2}}\right] .
$$

where $\bar{X}$ denotes a flux surface average of $X$ and again $X^{\prime}$ denotes a derivative, or partial derivative, of $\mathrm{X}$ in $r$. Furthermore, $\delta=\Delta^{\prime} \sin \Theta /\left(B_{0} R_{0}^{2}\right)$ is negligible in the calculation for the toroidal drift for moderate plasma pressure and conventional aspect ratio. Following the regime employed in Connor et al [4] we henceforth assume that the effect of finite beta on the equilibrium is only seen through locally large radial gradients in the pressure. Hence $r\left|p_{\perp}^{\prime}\right| \gg p_{\perp}$ and $r\left|p_{\|}^{\prime}\right| \gg p_{\|}$so that $r \Delta^{\prime \prime}>>\Delta^{\prime}$ and $\left|r \sigma^{\prime}\right| \gg|\sigma-1|$. Thus terms involving $\sigma^{\prime}$ and $\tau$ remain, but $\sigma$ is replaced by unity. Furthermore, noting that $r I^{\prime} \gg I$ we obtain the identity:

$$
-\left.\frac{g q+I}{\sigma^{2}} \frac{\partial \sigma}{\partial \psi}\right|_{\Theta}+\frac{d I}{d \psi}+q \frac{d g}{d \psi}=-\frac{R_{0} q^{2}}{r B_{0}^{2}}\left(\frac{\partial p_{\perp}}{\partial r}-\frac{\partial p_{\|}}{\partial r}+\frac{\partial \overline{p_{\|}}}{\partial r}\right) .
$$

where we note that $\partial \overline{p_{\|}} / \partial r-\partial p_{\|} / \partial r$ can be neglected in the above because the poloidal variation in $p_{\|}$is only non-negligible when $p_{\perp} \gg p_{\|}$[10]. Finally, we can now use the large aspect ratio expression $\sigma^{2} B^{2} \approx g^{2} / R^{2}+I^{2} / r^{2}$ to reveal the leading order identity:

$$
\left.\frac{\partial B}{\partial \psi}\right|_{\Theta}=-\frac{q}{r R_{0}}\left(\left.\frac{\partial R}{\partial r}\right|_{\Theta}-\frac{\alpha_{\perp}}{2 q^{2}}\right)
$$

which together with the definition of the poloidal angle $\theta$, for which $R=R_{0}-\Delta+r \cos \theta$, $Z=r \sin \theta$ and $d \Theta=d \theta R B^{2} \overline{J_{c}} B_{0}^{-1}(g+I / q)^{-1}$, with $\overline{J_{c}}=1-\Delta^{\prime} \cos \theta$, we obtain

$$
\langle\dot{\phi}\rangle=\tau_{b}^{-1} \oint d \theta q+\tau_{b}^{-1} \oint d \theta \frac{q^{2}}{r \Omega_{c}}\left[\left.\left(\frac{\mu B_{0}}{m v_{\|}}+v_{\|} \tau\right) \frac{\partial R}{\partial r}\right|_{\Theta}+\left(\frac{\mu B_{0}}{m v_{\|}}+v_{\|}(\tau-1)\right) \frac{\alpha_{\perp}}{2 q^{2}}\right] .
$$

Notice that the first term in Eq. (18) should be expanded to first order in the ratio of transit velocity and drift velocity. This is done by expanding $q(r)$ about its transit average value, i.e. we write $q(r)=q(\langle r\rangle)+(r-\langle r\rangle) d q / d r$. For trapped particles orbits, $\oint d \theta q(\langle r\rangle)=0$, which means that we are left with only the first order term in the expansion. This is obtained by noting that $r-\langle r\rangle=\int_{\langle t\rangle}^{t} d t \dot{r}=\left(q / r B_{0}\right) \int_{\langle\Theta\rangle}^{\Theta} d \Theta \dot{\psi} / \dot{\Theta}$, where $\langle t\rangle$ is the time, and $\langle\Theta\rangle$ the angle, at which $r$ is at its orbit averaged value $\langle r\rangle$. Now, from Eqs. (15b) and (15c) (and conservation properties of $\mu$ and $E$ ) we have approximately $\dot{\psi} / \dot{\Theta}=-g \partial / \partial \Theta\left(v_{\|} / \sigma B\right)$ so that $r-\langle r\rangle=\left(q / r B_{0}\right)\left(v_{\|}(\Theta) R(\Theta)-\right.$ $\left.v_{\|}(\langle\Theta\rangle) R(\langle\Theta\rangle)\right)$, and hence for trapped particles

$$
\oint d \theta q=\frac{q^{2} s}{\epsilon \Omega_{c} r} \oint d \theta v_{\|} \text {. }
$$

Finally, the last remaining pressure effect (the local shear effect) is contained in $\partial R /\left.\partial r\right|_{\Theta}$. We use $\cos \theta=\cos \Theta-\left(\epsilon+\Delta^{\prime}\right) \sin \Theta$, and the major radius written in terms of the Boozer angle $\Theta$ is $R=R_{0}-\Delta+r \cos \Theta-r\left(\epsilon+\Delta^{\prime}\right) \sin ^{2} \Theta$, to give $\partial R /\left.\partial r\right|_{\Theta}=\cos \theta-\Delta^{\prime}-\left(\epsilon+r \Delta^{\prime \prime}\right) \sin ^{2} \theta$. Again we employ $r \Delta^{\prime \prime} \gg \Delta^{\prime}$, and to leading order in $\epsilon$ we finally arrive at

$$
\langle\dot{\phi}\rangle=\tau_{b}^{-1} \oint d \theta \frac{q^{2}}{r \Omega_{c}}\left[\frac{s}{\epsilon} v_{\|}+\left(\frac{\mu B_{0}}{m v_{\|}}+v_{\|} \tau\right)\left(\cos \theta-r \Delta^{\prime \prime} \sin ^{2} \theta\right)-\left(\frac{\mu B_{0}}{m v_{\|}}+v_{\|}(\tau-1)\right) \frac{\alpha_{\perp}}{2 q^{2}}\right],
$$


with $\tau_{b}=q R_{0} \oint d \theta / v_{\|}$. Finally we employ the pitch angle $k^{2}=\left[\left(1-\lambda B_{0}(1-\epsilon)\right] /\left(2 \lambda B_{0} \epsilon\right)\right.$, where $\lambda=\mu B_{0} / E$, so that $v_{\|}^{2}=4 E \epsilon\left[k^{2}-\sin ^{2}(\theta / 2)\right]\left[1+\epsilon\left(2 k^{2}-1\right)\right]^{-1}$, and the bounce angle $\theta_{b}=2 \arcsin (k)$. For trapped particles the terms proportional to $v_{\|} \tau$ and $v_{\|}(\tau-1)$ are not leading order in $\epsilon$, so that we can write approximately,

$$
\langle\dot{\phi}\rangle=\frac{q E}{r R_{0} m \Omega_{c}}\left[G_{1}\left(k^{2}\right)+2 s G_{2}\left(k^{2}\right)-r \Delta^{\prime \prime} G_{3}\left(k^{2}\right)-\frac{\left\langle\alpha_{\perp}\right\rangle\left(k^{2}, r\right)}{2 q^{2}}\right],
$$

where in the limit of $r p_{\perp}^{\prime} \gg p_{\perp}$ and $r p_{\|}^{\prime} \gg p_{\|}$, and for values of $\alpha$ much larger than $\epsilon$ we have

$$
r \Delta^{\prime \prime}=\bar{\alpha},
$$

where we identify

$$
\bar{\alpha} \equiv-\frac{R_{0} q^{2}}{B_{0}^{2}}\left(\frac{1}{2 \pi}\right) \int_{0}^{2 \pi}\left(\frac{\partial p_{\|}}{\partial r}+\frac{\partial p_{\perp}}{\partial r}\right) d \theta .
$$

Furthermore, $G_{1}, G_{2}, G_{3}$ and $\left\langle\alpha_{\perp}\right\rangle\left(k^{2}, r\right)$ can be written in terms of elliptic integrals $K\left(k^{2}\right)=\int_{0}^{\pi / 2} d \omega\left[1-k^{2} \sin ^{2} \omega\right]^{-1 / 2}$ and $E\left(k^{2}\right)=\int_{0}^{\pi / 2} d \omega\left[1-k^{2} \sin ^{2} \omega\right]^{1 / 2}$ to give,

$$
\begin{aligned}
G_{1}\left(k^{2}\right) & =2 E\left(k^{2}\right) / K\left(k^{2}\right)-1 \\
G_{2}\left(k^{2}\right) & =2 E\left(k^{2}\right) / K\left(k^{2}\right)+2\left(k^{2}-1\right) \\
G_{3}\left(k^{2}\right) & =\frac{4}{3}\left[\left(2 k^{2}-1\right) E\left(k^{2}\right) / K\left(k^{2}\right)+\left(1-k^{2}\right)\right] . \\
\left\langle\alpha_{\perp}\right\rangle\left(k^{2}, r\right) & =\frac{\int_{0}^{\pi / 2} d \omega \alpha_{\perp}(r, \omega)\left[1-k^{2} \sin ^{2} \omega\right]^{-1 / 2}}{K\left(k^{2}\right)},
\end{aligned}
$$

where $\alpha_{\perp}(r, \theta)$ is defined in Eq. (9), and $\omega=\arcsin [\sin (\theta / 2) / k]$, such that $\alpha_{\perp}(r, \theta)$ can be mapped in Eq. (23) using $\theta=2 \arcsin (k \sin \omega)$.

Finally, we summarise the finite beta effects in $\langle\dot{\phi}\rangle$ shown in Eq. (20). We recognise the term involving $r \Delta^{\prime \prime}$ as the local shear effect first highlighted by Connor et al [4], and by solving the equilibrium numerically in this paper, we find that it is relatively insensitive to pressure anisotropy for fixed $\bar{\alpha}$. The latter statement is clearly true also when solving for $\Delta$ using the anisotropy generalised Grad-Shafranov equation of Ref. [10], which obtains the result of Eq. (21). The other pressure term in Eq. (20) is proportional to $\left\langle\alpha_{\perp}\right\rangle$, and is a generalisation of the diamagnetic effect derived in Ref. [4]. This term is sensitive to anisotropy in two ways: firstly, for plasmas with parallel anisotropy, the effect of pressure (parallel pressure) through the diamagnetic effect is negligible, and secondly, perpendicular anisotropy creates a strong poloidal dependence in $p_{\perp}$, which ultimately influences $\left\langle\alpha_{\perp}\right\rangle$. In particular, the orbit averaging of $\alpha_{\perp}$ is weighted most heavily at the trapped ion turning points, thus giving

$$
\left\langle\alpha_{\perp}\right\rangle \equiv \frac{\oint \alpha_{\perp}(r, \theta) d t}{\tau_{b}} \approx \alpha_{\perp}\left(r, \theta_{b}\right)
$$

with $\theta_{b}=2 \arcsin (k)$ the bounce angle. The poloidal profile of $\alpha_{\perp}$ is determined by the location of RF heating, and this therefore influences the variation of $\langle\dot{\phi}\rangle$ with pitch angle (e.g. $\left.\theta_{b}\right)$. 
Having derived an analytical expression for the toroidal drift, we now investigate the dependence of the drift on pressure. For what follows, let us re-write Eq. (20) in a more convenient form for discussing the numerical results:

$$
\omega_{D}=\omega_{D}^{\beta=0}-\omega^{\prime}\left(r \Delta^{\prime \prime} G_{3}\left(\theta_{b}\right)+\frac{\alpha_{\perp}\left(r, \theta_{b}\right)}{2 q^{2}}\right)
$$

where $\omega_{D}^{\beta=0}$ includes all the terms not related to pressure and $\omega^{\prime}=q E / r R_{0} m \Omega_{c}$. It is important to note that these analytical expressions are not used for the numerical results shown later, which are entirely obtained using a numerical equilibrium and integrating the orbit equations (15a) - (15d) over time. Nevertheless, Eqs. (20) and (25) enable us to separate and identify the contrasting effects of pressure on the magnetic drift for the obtained numerical results, and we will refer to them in what follows.

\subsection{Local shear effect}

In the following, a JET-like equilibrium was used with a major radius of $R_{0}=3.16 \mathrm{~m}$, edge minor radius $a=1 \mathrm{~m}$, elongation $\kappa_{a}=1.4$ and triangularity $\delta_{a}=0.4$. The $q$-profile was chosen to be $q=q_{0}+\Delta q\left(r / r_{1}\right)^{2}$, with $q_{0}=0.7, \Delta q=1-q_{0}$ and $r_{1}=0.4$. For the calculation of the drift frequency, we followed a test ion of $10 \mathrm{eV}$ close to the location of maximum pressure gradient by integrating Eqs. (15a) - (15d) over time. We chose a rather low energy in order to assure negligible banana orbit width. At each time step, local $\alpha$ and $\alpha_{\perp}$ are calculated and in the end averaged over the total simulation time. This gives a weighted orbit average of $\alpha_{\perp}$, counting the turning points more heavily than the rest of the banana orbit, since the particle spends more time at those points than elsewhere. Mathematically, this corresponds to Eq. (24). At the end of the simulation, the average toroidal drift frequency is calculated using the trivial relation

$$
\langle\dot{\varphi}\rangle=\frac{\varphi_{e}-\varphi_{i}}{t_{t o t}}
$$

where $\varphi_{e}$ is the value of the toroidal angle $\varphi$ at the last passing of the midplane, $\varphi_{i}$ the toroidal angle at the first passing and $t_{t o t}$ the total simulation time in-between the first and the last passing of the particle at the midplane. Eq. (26) is used to calculate the toroidal drift frequency in all following plots.

We first concentrate on the local shear effect, which in Eq. (25) we recognise as the term proportional to $r \Delta^{\prime \prime}$. We compare four equilibria, one with $\beta=0$, and the other three having the same profile for $\bar{\alpha}$, as defined in Eq. (22), which at its peak (at $r / a=0.4$, where all the simulations are done) is $\bar{\alpha} \approx 1.03$. The latter three cases however differ with respect to their values of $T_{\perp} / T_{\|}$, and are shown in Fig. 3 to have $T_{\perp} / T_{\|}=1 / 10,1$ and 10 .

In each it is seen that the ICRH resonance location is chosen to pass close to the magnetic axis, i.e. $B_{c} \approx B_{0} \approx 3.1 T$. Fig. 3(c) demonstrates the clearest departure from isotropy. It is seen that the pressure peak is elongated along the resonant layer $B=B_{c}$. This is due to the fact that hot particle deposition at that location generates preferential banana orbits with tips close to $\theta= \pm 90^{\circ}$. Despite such dependencies with 


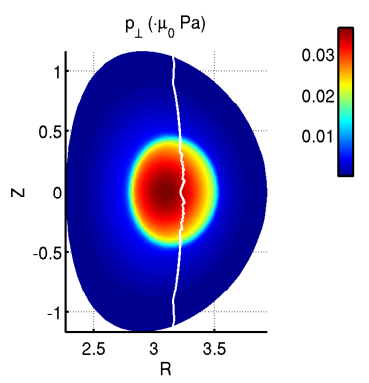

(a) $T_{\perp} / T_{\|}=1 / 10$.

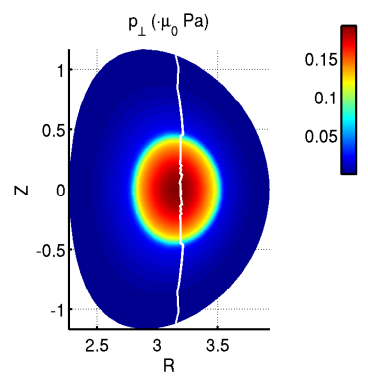

(b) $T_{\perp} / T_{\|}=1$.

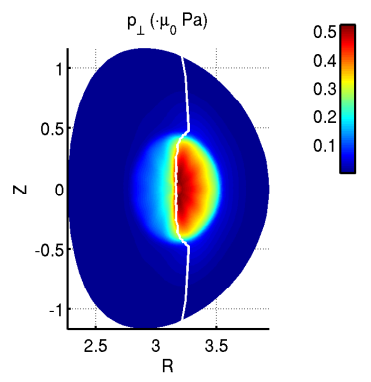

(c) $T_{\perp} / T_{\|}=10$.

Figure 3. The perpendicular pressure in the RZ plane for the different considered anisotropic cases. The white lines denote the locations of $B=B_{c}$.

respect to $\psi$ and $\theta$ in Fig. 3, it turns out that by keeping $\bar{\alpha}(r)$ the same for all cases in Fig. 3, the profile for the Shafranov shift is almost the same [10], as shown in Fig. 4(a), where we plot $r \Delta^{\prime \prime}$. The only contrasting profile is that of the case with zero $\beta$. It follows then that the local shear effect should lead to the same offset in $\omega_{D}$ for the three cases relative to the $\beta=0$ case. The magnetic drift frequencies corresponding to the four equilibria, normalised to $\omega^{\prime}$, are shown in Fig. 4(b). It follows that the differences between the lower three curves can be identified with the offset due to the diamagnetic effect (last term in Eq. (25)), and will be described in the next section. We can however most easily identify the local shear effect by comparing the top two curves in Fig. 4(b), i.e. the case with zero $\beta$, and the case with $T_{\perp} / T_{\|}=1 / 10$. The reason for this is that both of the latter cases have small $\alpha_{\perp}$, the corresponding diamagnetic effect giving a small and almost constant offset for all bounce angles, the value being that of the differences of the curves at $\theta_{b}=0^{\circ}$ or $\theta_{b}=180^{\circ}$ (since then $G_{3}=0$ ). Hence, the local shear effect is seen to reduce the drift for intermediate pitch angles, as is clear if one plots $G_{3}$ as a function of $\theta_{b}$. If at the same time $\alpha_{\perp}$ is sufficiently large, the local shear effect can enable a wider region of pitch angle space where the direction of $\omega_{D}$ is reversed. This is the case for the isotropic finite $\beta$ curve, and the $T_{\perp} / T_{\|}=10$ curve in Fig. 4(b). Nevertheless, we see that the finite $\beta$ effect in the perpendicular anisotropy case and the isotropic finite $\beta$ case is dominated by the diamagnetic effects, and these are described next.

\subsection{Diamagnetic effect}

Figure 5 shows the same frequencies as before, though not including the $\beta=0$ case, but now compared to the values of $\left\langle\alpha_{\perp}\right\rangle$ instead of $r \Delta^{\prime \prime}$. One can clearly see a bump appearing in the drift frequency for the case of perpendicular anisotropy $\left(T_{\perp} / T_{\|}=10\right)$, located at same bounce angle as the maximum of $\left\langle\alpha_{\perp}\right\rangle$. Also, $\omega_{D}$ is considerably higher for parallel anisotropy $\left(T_{\perp} / T_{\|}=1 / 10\right)$, where $\left\langle\alpha_{\perp}\right\rangle$ is much lower. Note that for the three different cases $T_{\perp} / T_{\|}=10,1$ and 1/10, the flux surface averaged parameter $\bar{\alpha}$ at $r / a=0.4$ was a constant value $\bar{\alpha} \approx 1.03$. From this, three different conclusions can 


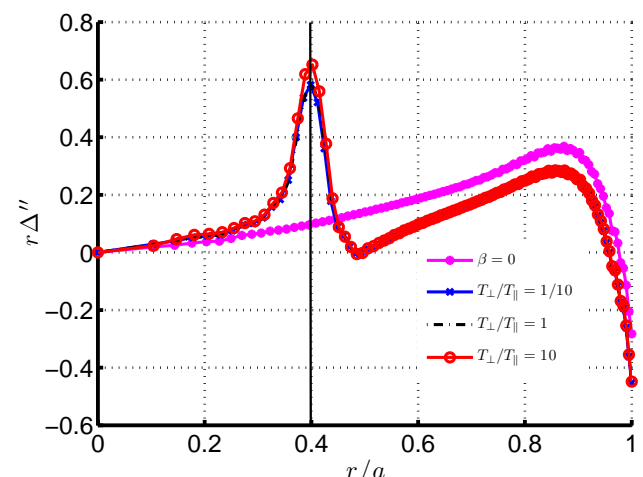

(a) Profiles of $r \Delta^{\prime \prime}$ for the four equilibria.

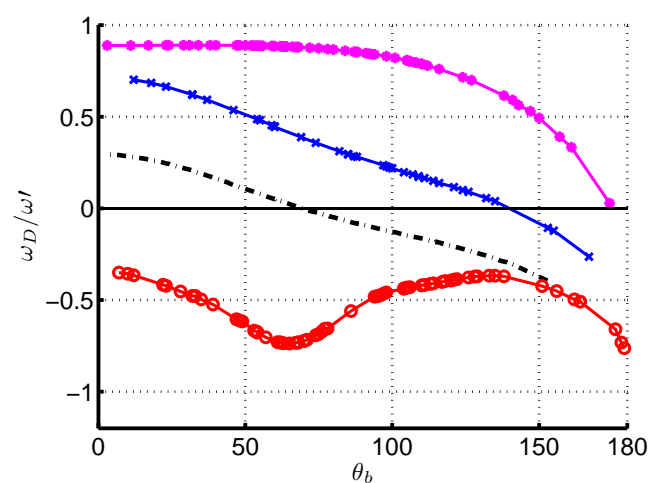

(b) The precession drift frequencies as functions of the bounce angle.

Figure 4. The toroidal precession drift frequencies are different from one another, even if the local shear has the same effect in all cases. The line in (a) shows the radial location of the orbits. Starred $(*)$ and magenta: $p=0$, crosses $(\mathrm{x})$ and blue: $T_{\perp} / T_{\|}=1 / 10$, dots and black: $T_{\perp} / T_{\|}=1$, circles (o) and red: $T_{\perp} / T_{\|}=10$.

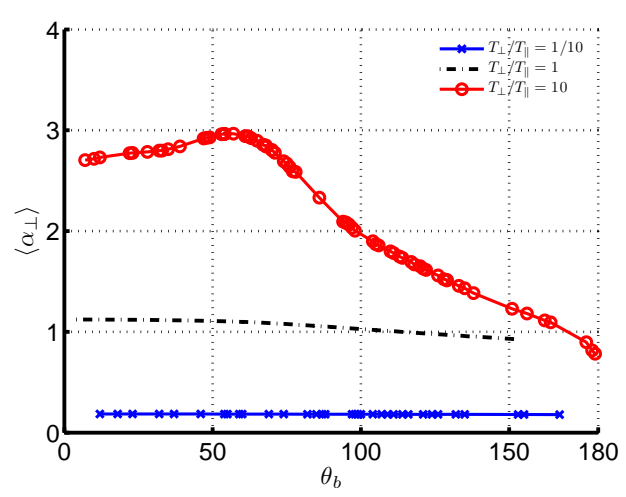

(a) $\alpha_{\perp}$ as a function of the bounce angle.

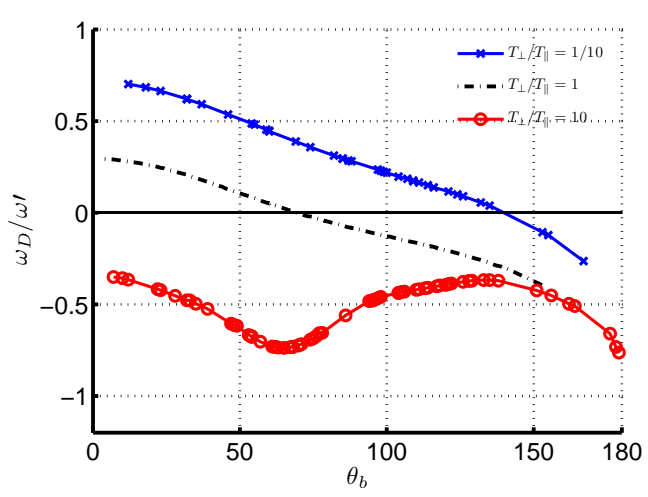

(b) Toroidal magnetic drift frequency

Figure 5. The toroidal drift frequency depends on the parameter $\alpha_{\perp}=$ $-2 R_{0} q^{2} / B_{0}^{2}\left(p_{\perp}^{\prime}\right)$, which has a strong poloidal dependence.

be drawn: First, the diamagnetic effect is more important than the local shear effect for $T_{\perp} / T_{\|} \geq 1$ and $q \sim 1$. Second, the diamagnetic effect can also lower the toroidal precession drift frequency of deeply trapped particles, such that the frequency can be negative for all pitch angles. Third, anisotropy can introduce an important poloidal dependence of $\omega_{D}$ through a poloidal dependence of $\alpha_{\perp}$, i.e. of the perpendicular pressure and its gradient.

With off-axis heating, it is possible to shift the maximum pressure gradient in the RZ plane. Fig. 6 shows the pressure surfaces for high field side (HFS) and low field side (LFS) heating. As stated above, the heating location can be chosen via the value of the parameter $B_{c}$ in the equilibrium code VMEC. Note that the peak of the pressure profile is not exactly at the locations of $B=B_{c}$. For all of the equilibria used in this article, a sharp pressure gradient was imposed at the radial location $r / a=0.4$. Outside this 


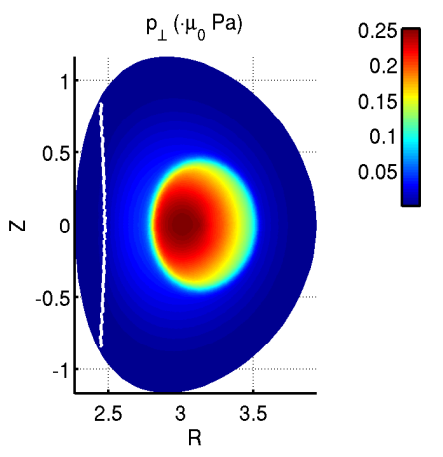

(a) Pressure surfaces for HFS heating.

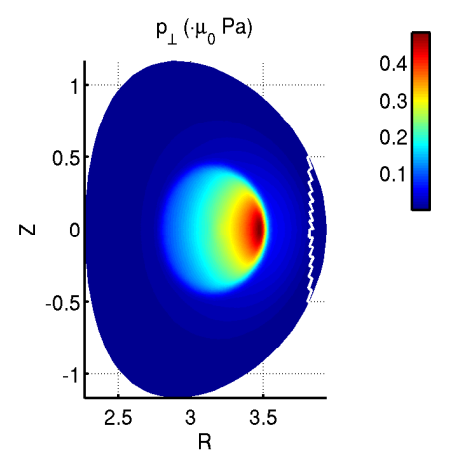

(b) Pressure surfaces for LFS heating.

Figure 6. $p_{\perp}$ in the RZ plane with resonant heating on (a) the HFS and (b) the LFS. In both $T_{\perp} / T_{\|}=10$, and they can be compared with the reference equilibrium shown in Fig. 1(a), which has the resonant heating through the magnetic axis.

surface, the pressure is very low. The effect of LFS heating on the poloidal variation of $p_{\perp}$ is very strong, since in that case the deeply trapped particle fraction is higher than for HFS heating, where more barely trapped particles are created. More barely trapped particles tend then to average out a poloidal variation in $p_{\perp}$. The shifting of the pressure peak towards the inboard side (HFS heating) results in a shift in the maximum pressure gradient towards $\theta=\pi$, whereas the shifting of the pressure peak towards the outboard side (LFS heating) results in a shift in the maximum pressure gradient towards $\theta=0$. As a result, one can see in Fig. 7 that the bump in precession drift frequency is shifted in the exact same way. For LFS heating, the toroidal drift frequency is even negative for deeply trapped particles, whereas it goes negative for large bounce angle in the case of HFS heating.

With these studies it becomes evident that the important parameter for determining the magnetic precession drift frequency is not the conventional diamagnetic term proportional to $\alpha$, but rather its perpendicular analogue $\alpha_{\perp}\left(\theta_{b}\right)$ weighted close to the bounce angle $\theta_{b}$. 


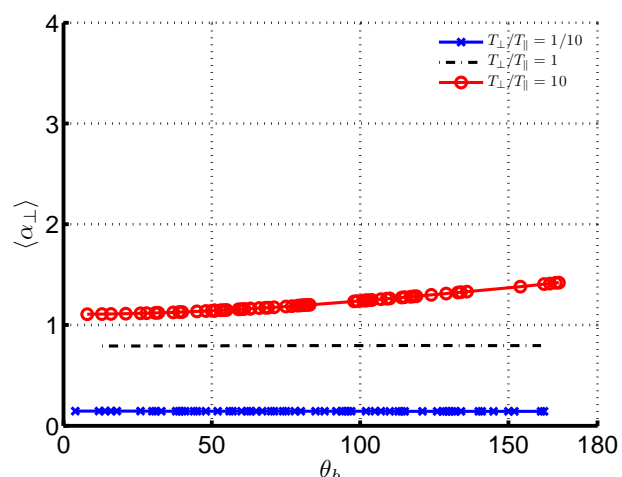

(a) HFS heating: $\left\langle\alpha_{\perp}\right\rangle$ as a function of the bounce angle.

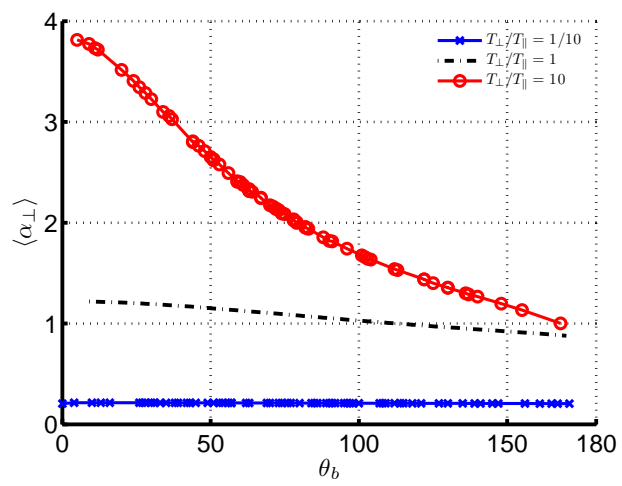

(c) LFS heating: $\left\langle\alpha_{\perp}\right\rangle$ as a function of the bounce angle.

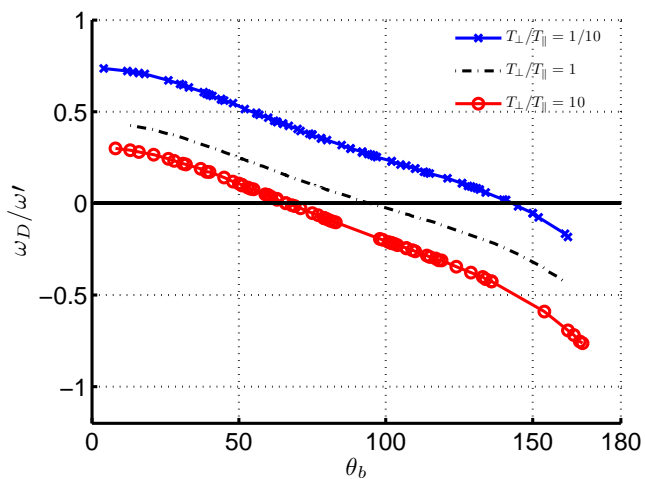

(b) HFS heating: Toroidal magnetic drift frequency

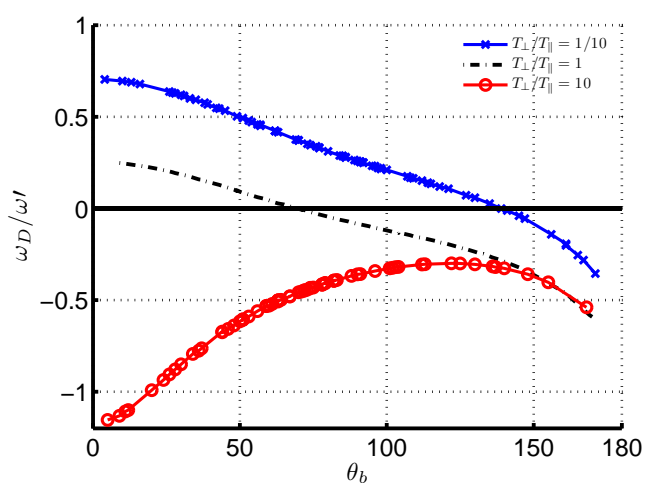

(d) LFS heating: Toroidal magnetic drift frequency

Figure 7. As the peak in $\left\langle\alpha_{\perp}\right\rangle$ shifts to smaller or larger bounce angles for respectively LFS or HFS heating, the form of the drift frequency changes accordingly. The dependence on $\theta_{b}$ should be compared with the on-axis heating case of Fig. 5. 


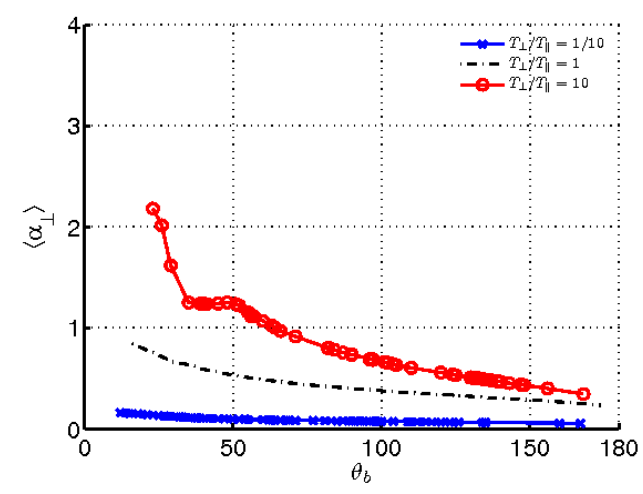

(a) LFS heating with wide banana orbits. Bounce averaged $\left\langle\alpha_{\perp}\right\rangle$ as a function of the bounce angle.

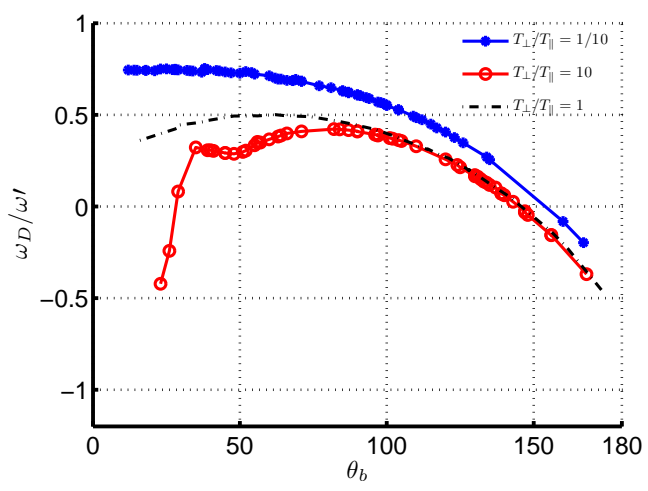

(b) LFS heating with wide banana orbits. Toroidal magnetic drift frequency

Figure 8. Larger particle energies, and wider orbits, modify the magnetic drift frequency in equilibria with highly localised pressure gradients.

\subsection{Large orbit width relative to gradient length scales}

If we now allow for an increase in the banana orbit width, we can study the case where the characteristic length scale of the pressure gradient is of the order of the banana width, $L_{p} \sim \Delta r$. Fig. 8 shows the effect on the case shown in Fig. 7 (LFS heating), but this time with an energy of $300 \mathrm{keV}$, such that the banana width is about the same as the pressure gradient length. The strong dependence on the bounce angle for deeply trapped particles can be understood as follows: For less deeply trapped orbits (higher $\theta_{b}$ ), the particle sees a lower average pressure gradient, since its large banana width leads it into regions with lower $\alpha_{\perp}$, whereas deeply trapped particles do not see these regions, since their orbit widths are much smaller, and thus their orbit averaged $\left\langle\alpha_{\perp}\right\rangle$ is considerably larger. A second effect is the poloidal variation of $\alpha_{\perp}$ with perpendicular anisotropy (as already seen above), which is responsible for the plateau in $\left\langle\alpha_{\perp}\right\rangle$ (red line) between 30 and 60 degrees. It is interesting to note that while the greatest effect is for perpendicular anisotropy, it is still considerable for the isotropic case, where $\alpha_{\perp}$ more than doubles.

\section{Tear drop orbits and the poloidal variation of the magnetic field strength}

A particle is trapped if its parallel velocity vanishes due to high magnetic field strength, as can be seen in the relation

$$
\frac{v_{\|}}{v}= \pm \sqrt{1-\frac{\mu B}{E}}
$$

In a large aspect ratio isotropic plasma, the poloidal variation of $B$ is given by $B \sim 1 / R$, so that trapping is up-down-symmetric, and thus the particle passes through the outer midplane $\theta=0$. If however $B$ is non-monotonic with respect to $\theta$, particles can be locally trapped in the corresponding magnetic well. Two examples of non-monotonic $B$ 


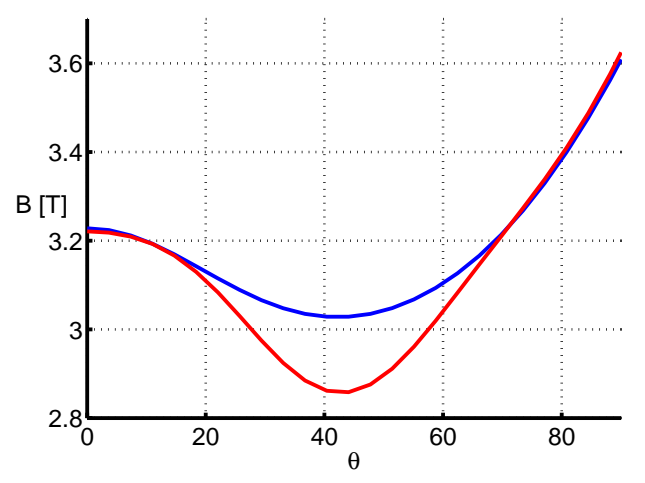

Figure 9. Poloidal dependence of the pressure results in a stronger poloidal dependence of the magnetic field strength. Here, $T_{\perp} / T_{\|}=1, T_{\perp} / T_{\|}=10$.

profiles are shown in Fig. 9 and will be described later. The trajectories of such trapped particles differ from conventional trapped orbits in that they stay either on the upper $(0<\theta<\pi)$ or lower half $(\pi<\theta<2 \pi)$ of the plasma, and thus do not pass through the outer midplane. Such kinds of orbits are then called tear drop orbits, because of their trajectory in the RZ plane (see Fig. 11(a)). In isotropic plasmas, such orbits occur only in tight aspect ratio, because the toroidal and poloidal magnetic fields are of the same order. Nevertheless we point out here that the localised trapping can be enhanced with perpendicular pressure anisotropy. This can be seen by referring to Eqs. (7) and (8), and noting that the toroidal component of the field strength depends on $\theta$ through the perpendicular pressure $p_{\perp}(r, \theta)$ when $T_{\perp} / T_{\|} \gg 1$ (in addition to the conventional $1 / R$ dependence).

Having the possibility of poloidal dependence in the pressure and magnetic field due to anisotropy, we can enhance the variation of the magnetic field strength in the poloidal angle, as e.g. shown in the isotropic and anisotropic cases of Fig. 9. The corresponding perpendicular pressure for the latter two equilibria are shown in Fig. 10. In this part of our work, we considered a tight aspect ratio configuration with $R_{0}=1.16 \mathrm{~m}, a=0.9 \mathrm{~m}$ and an elongation of $\kappa_{a}=2.5$ at the edge. This was chosen such that tear drop orbits already can occur in the isotropic case as described above. The triangularity was zero and the safety factor profile unchanged from the previous section. For both the anisotropic and isotropic case, the volume averaged beta $\langle\beta\rangle \approx 1 \%$ was kept constant and the flux averaged $\bar{\alpha}$ was similar with $\bar{\alpha} \approx 0.68$ for $T_{\perp} / T_{\|}=10$ and $\bar{\alpha} \approx 0.75$ for $T_{\perp} / T_{\|}=1$. However, as can be seen in Fig. 10, due to the LFS deposition employed, there are two sharp peaks in the anisotropic case compared to the isotropic equilibrium. These two sharp peaks are responsible for a deepened magnetic well shown Fig. 9. In the latter figure, the curves show the magnetic field strength as a function of poloidal angle at the radial location of the pressure peaks. The blue (upper) curve is the isotropic and the red (lower) curve is the anisotropic case. With the deepening of the magnetic well, i.e. lowering the minimum field, trapped particles at the well minimum achieve higher parallel velocities according to Eq. (27). Thus the particle 


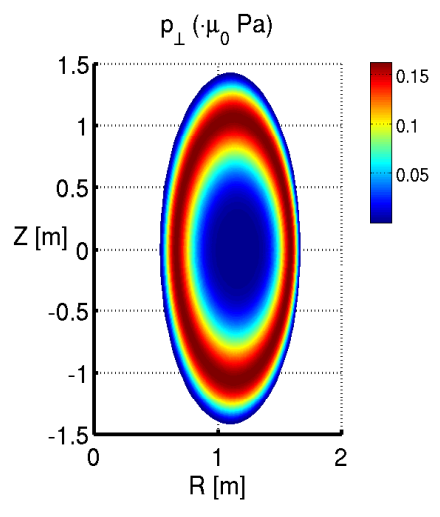

(a) Isotropic case: pressure is a flux surface quantity.

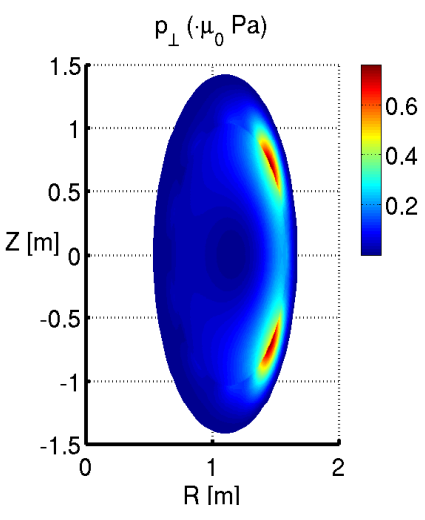

(b) Anisotropic case: pressure has now a poloidal dependence.

Figure 10. Strong poloidal dependence of the pressure due to anisotropy and off-axis heating.

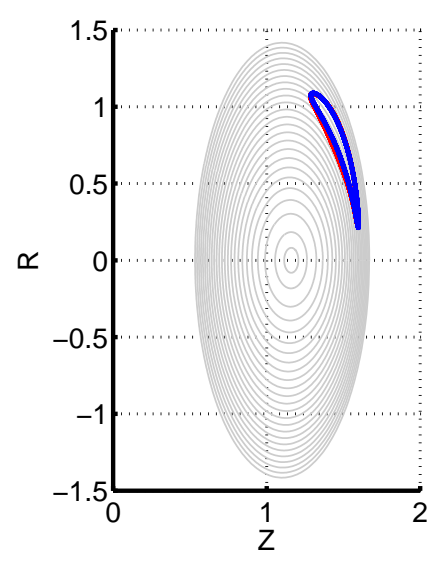

(a) Tear drop orbit in RZ: almost no difference.

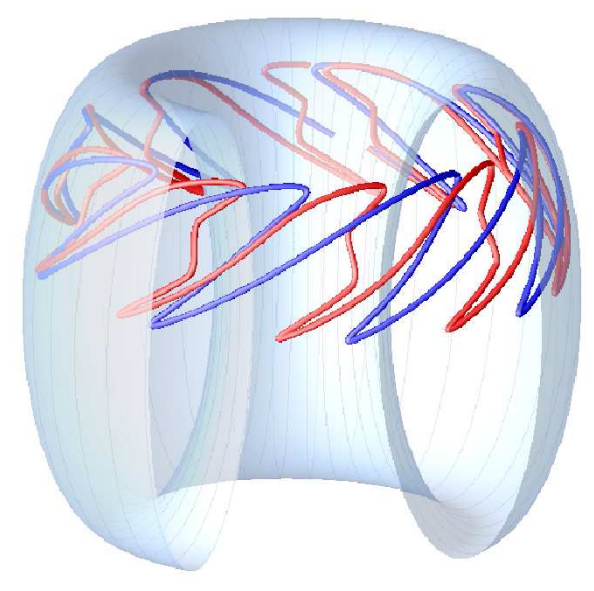

(b) In 3D: one can clearly see a difference in toroidal direction.

Figure 11. The deepened magnetic well has an impact mainly on the toroidal direction.

orbits in isotropic and anisotropic equilibria are different in three dimensional space, and especially in the toroidal direction, as shown in Fig. 11. Here we show that the difference in the RZ plane (tear drop orbits) is rather small, whereas it is considerable in the toroidal angle, as shown by the s-like trajectory of the orbit in the 3D plot of Fig. 11(b) (red curve). Note that the orbits were chosen in such a way that the turning points are at the same location (poloidally and radially) in both cases. 


\section{Conclusions}

In this work, we have investigated the impact of pressure anisotropy on both tokamak equilibria and single particle orbits. In particular, single particle orbits were used in order to compute the toroidal precession drift of test particles in such new anisotropic equilibria. As stated in earlier work, collisionless trapped-electron modes, fishbones and other instabilities can be stabilised through diamagnetism [1,4] and local as well as global shear $[2,4]$ by inverting the toroidal precession drifts of trapped particles. We note that the diamagnetic effect is proportional to the radial pressure gradient, the local shear to the second derivative of the Shafranov shift and the global shear to the radial derivative of the safety factor. However, as Connor et al. [4] stressed before, a consistent treatment of finite $\beta$ effects from energetic ions is only possible if an anisotropic pressure equilibrium is taken into account. Indeed, we found in this article that the diamagnetic effect is not due to the total pressure derivative, but exclusively the derivative of the perpendicular component $p_{\perp}$. Thus, the diamagnetic effect is most important for perpendicular anisotropy $T_{\perp} / T_{\|}>1$, where it is much more important than the local and global shear effects when $q \sim 1$. Note that perpendicular anisotropy occurs e.g. in ICRH heated plasmas, where the ions are heated perpendicularly. At the same time, the diamagnetic effect has a much weaker impact on the precession drift in the case of parallel anisotropy $T_{\perp} / T_{\|}<1$, as is the case for e.g. NBI heating. Furthermore, pressure anisotropy opens the way for poloidal pressure dependence, such that the pressure is not a flux surface quantity anymore but can have a strong variation in $\theta$ direction. This poloidal dependence in the pressure then induces a strong dependence of the toroidal precession drift frequency in perpendicularly anisotropic equilibria, such that the drift can be inverted in a large region of pitch angle space. Also, we have shown how the pitch angle dependence of the precession drift frequency changes as the banana orbit width becomes similar to the pressure gradient length.

The poloidal distribution of the hot particle pressure is governed by the deposition layer of the hot particles, defined by $B=B_{c}$. Indeed, we have shown that the $\theta$ dependence is stronger for LFS than HFS heating, since in the latter case barely trapped particles are preferentially created, adding to the pressure over the complete flux surface and thus averaging out poloidal dependence, whereas for LFS heating deeply trapped particles are deposited, staying close to $\theta=0$ during their entire evolution. Another effect of the poloidal pressure dependence is the deepening of magnetic wells in poloidal direction, giving rise to so called tear drop orbits. These are orbits where the trapped particles do not cross the midplane at $\theta=0$, but stay either on the upper $(0<\theta<\pi)$ or lower half $(\pi<\theta<2 \pi)$ of the plasma. Two such orbits are shown in order to illustrate the effect of the deepening of the poloidal well. While there is very little difference between the orbits in the RZ plane for the isotropic and the anisotropic equilibrium cases, the differences become clear in the third (toroidal) direction. 


\section{Acknowledgments}

This work was supported in part by the Swiss National Science Foundation. We thank Dr. S.P. Hirshman for the use of the VMEC code. 
[1] M.N. Rosenbluth and M.L. Sloan. Phys. Fluids, 14:1725, 1971.

[2] M.A. Beer, G.W. Hammett, G. Rewoldt, E.J. Synakowski, M.C. Zarnstorff, and W. Dorland. Phys. Plasmas, 4:1792, 1997.

[3] Y. Wu, C.Z. Cheng, and R.B. White. Phys. Plasmas, 1:3369, 1994.

[4] J.W. Connor, R.J. Hastie, and T.J. Martin. Nucl. Fusion, 23:1702, 1983.

[5] W.A. Cooper, S.P. Hirshman, S. Merazzi, and R. Gruber. Comp. Phys. Comm., 72:1, 1992.

[6] D.V. Anderson, W.A. Cooper, G.Y. Fu, M. Gengler, R. Gruber, S. Merazzi, and U. Schwenn. Supercomp. Rev., 3:29, 1991.

[7] W.A. Cooper, J.P. Graves, S.P. Hirshman, T. Yamaguchi, Y. Narushima, S. Okamura, S. Sakakibara, C. Suzuki, K.Y. Watanabe, H. Yamada, and K. Yamazaki. Nucl. Fusion, 46:683, 2006.

[8] J. P. Graves, K. I. Hopcraft, R. O. Dendy, R. J. Hastie, K. G. McClements, and M. Mantsinen. Sawtooth evolution during jet ion-cyclotron-resonance-heated pulses. Phys. Rev. Lett., 84(6):1204-1207, Feb 2000.

[9] J.P. Graves, W.A. Cooper, S. Coda, Eriksson L.-G., and T. Johnson. Joint Varenna-Lausanne International Workshop. AIP Conference Proceedings, 871:350, 2006.

[10] N. A. Madden and R. J. Hastie. Nucl. Fusion, 34:519, 1994.

[11] C.M. Roach, J.W. Connor, and S. Janjua. Plasma Phys. Control. Fusion, 37:679, 1995.

[12] C. Bourdelle, G.T. Hoang, X. Litaudon, C.M. Roach, and T. Tala. Nucl. Fusion, 45:110, 2005.

[13] W.A. Cooper, J.P. Graves, M. Jucker, and M.Yu. Isaev. Phys. Plasmas, 13:092501, 2006.

[14] H. Grad. Phys. Fluids, 9:498, 1966.

[15] G.A. Cooper, M. Jucker, W.A. Cooper, J.P. Graves, and M.Yu. Isaev. Phys. Plasmas, 14:102506, 2007.

[16] O. Fischer, W.A. Cooper, M.Yu. Isaev, and L. Villard. Nucl. Fusion, 42:817, 2002.

[17] R.B. White and M.S. Chance. Phys. Fluids, 27:2455, 1984.

[18] R.B. White, A. H. Boozer, and R. Hay. Phys. Fluids, 25:575, 1982. 